\title{
Plandf's Rommentar
}

\section{zum \\ Bïrgerlichen (Bejerbutd) \\ nebjt Cinführungsgejeg.}

Serausgegeben bon

Dr. E. Etrohal,

orbentltcuer profefior in Lelpaig,

in Berbinbung mit

Dr. F. $\mathfrak{A n d o r e ́ , ~}$ orb. Profeffor,

D. Breiff,

wirtl. Geb. Ober. Sujtizrat, oberlanbesgertatspräpent,

Dr. H. Giber,

orb. Profeftor,
E. Brobmann,

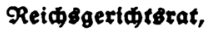

Dr. F. Flad,

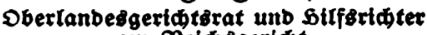
am Reidsgertat,

Dr. P. Rnofe, Dr. A. Mendelzjohn Bartholdy, orb. Sonorar.Profefior, orb. Profefinor,

Dr. D. Etreffer,

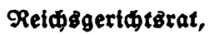

Dr. . v. $\mathfrak{U} \mathfrak{u} \mathfrak{z} \mathfrak{n} e \mathfrak{r}$,

Genatßpräftbent.

\author{
I. $\mathfrak{B a n d}$. \\ Ulfgemeiner Teil (\$§ 1-240).
}

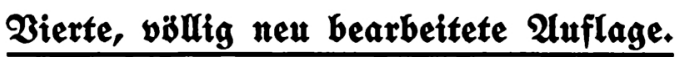

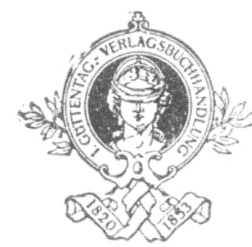

Berlin 1913.

3. (Guttentag, $\mathfrak{B} \mathfrak{e r l a g} \mathfrak{g} \mathfrak{b} \mathfrak{u} \mathfrak{b} \mathfrak{a} \mathfrak{n} b \mathfrak{l} \mathfrak{u} \mathfrak{g}$, G. m. b. S. 
